\title{
The Analysis of GIS Defect Model Partial Discharge Test
}

\author{
Liqiang Liu ${ }^{1, *}$ and Jiao Wang ${ }^{2}$ \\ ${ }^{1}$ College of Electric Power, Inner Mongolia University of Technology, huhhot 010080, China \\ ${ }^{2}$ State Grid East Inner Mongolia Economic Research Institute, huhhot 010020, China \\ ${ }^{*}$ Corresponding author
}

\begin{abstract}
In order to achieve the pattern recognition of gasinsulated switchgear GIS defect types of partial discharge, in this paper, the analysis of gas discharge theory and ANSYS simulation software are used to qualitatively analyzed the defect type. Based on the PD characteristics caused by four typical insulation defects, this paper designed corresponding artificial physical discharge models to measure the voltage and to collect data. ANFIS is used to recognize the defect type, and the results show that the application of ANFIS for GIS partial discharge defect type recognition can achieve a satisfying results.
\end{abstract}

Keywords-pattern recognition; gas-insulated switchgear; partial discharge; adaptive fuzzy neural inference system

\section{INTRODUCTION}

GIS equipment is one of the most important equipment in power system and widely used. It is significant to make GIS insulation defect detection regarding safe and reliable operation of GIS ${ }^{[1-2]}$. When GIS insulation defect exist within the device, the internal partial discharge is likely to occur. Partial discharge signal contains a lot of insulation status information, through which can determine its insulating status. Partial discharge is the sign of equipment insulation deterioration and the main reason of insulation deterioration ${ }^{[3]}$. Therefore, GIS partial discharge detection is better than the other state detection method ${ }^{4]}$. Partial discharge detection methods are divided into non-electrical measuring and electrical measuring. Electrical measuring methods mainly include pulse current method and UHF method. UHF method has better anti-interference ability and positioning ability ${ }^{[5]}$.In this paper, UHF method, highly sensitive to kinds of defect type model is mainly used to collect data ${ }^{[6-8]}$. Non-electrical measuring methods mainly include ultrasonic method and SF6 gas decomposition component detection method ${ }^{[9-11]}$.

In high-voltage electrical equipment, SF6 is often used as an insulating medium. Gas discharge theories have complex origin, and early stage of streamer discharge is a good point of entry and has been got more and more attention. With the rapid development in computational power, to study gas discharge process using numerical simulation method has become a hot point ${ }^{[12-14]}$.

Currently, there are a lot of measures to recognize the PD ${ }^{[15-16]}$. ANFIS is widely used to conduct classification and recognition ${ }^{[17-18]}$, but in GIS research has not been found. This paper will combine the extracted UHF signals with ANFIS for GIS fault diagnosis.
A section of specialized design and manufactured GIS sample is applied for test research platform in this paper, and several typical insulation defect model with big possibility occurred during GIS production, transport, assembly and operation could be set up in this test platform. The partial discharge detecting methods are mainly using the electrical measures which are including the pulse current method and UHF method. The initial discharge voltage of defect models is tested and the signal samples of UHF are collected of insulation defects, which lay the foundation for the future gas component analysis.

\section{GAS DISCHARGE THEORY}

The basic theories of Gas discharge are streamer discharge theory and Townsend discharge theory. Townsend and streamer discharge theory are based on impacting ionization, and Townsend electron avalanche which is the premise condition of streamer discharge theory which has further consideration of photoionization effect, achieved secondary electron avalanche, progressed the development of streamer, completed breakdown.

\section{A. The Reason for Using SF6 Gas as Insulating Dielectric Gas}

One of the effective measures to improve the gas gap breakdown voltage is using high electric strength gas, and the gaseous compounds containing halogen element that is often called high electrical strength gas. Because it contains halogen elements, and the gas has strong electronegativity and large relatively molecular weight, thus the impact ionization ability is reduced. SF6 gas can be used as a good electrical highintensity gas, and the excitation energy of SF6 gas is small, but the ionization energy of SF6 gas is large, which is very difficult to ionized electrons of SF6 gas. SF6 gas is an electronegative gas, even if electrons exist, and SF6 gas atoms will capture an electron to form a negative ion. In the process of formation of the negative ions, the air gap of the electron is gradually reduced. Due to the formation of negative ions will hinder the development of the breakdown process, SF6 gas is an ideal gas as insulating dielectric gas so far. 


\section{B. Non-uniform Electric Field in the Breakdown (Short Gap)}

\section{1) Case to rod plate gap}

a) Non-self-sustaining discharge stage

When the rod has a positive polarity, positive space charge will be accumulated around the rod electrode, the electric field closely near the rod electrode is reduced, the electric field of outer space is slightly enhanced. The streamer is difficult to form near the rod electrode, so self-sustaining discharge which named corona discharge is difficult to form.

When the rod has a negative polarity, after the electrons of electron avalanche leave the strong electric field area, it will not cause ionization. Positive ions continue to move to rod electrode, and near rod electrode appeared more concentrated positive space charge, which make the electric field distorted. The electric field is enhanced near the rod electrode, so the self-sustaining discharge conditions are easy to meet, and easy to form streamer and corona discharge.

\section{b) The development stage of streamer}

When the rod has a positive polarity, the head of positively charge plasma of streamer weaken the plasma electric field, and strengthen the electric field of its head. Electric field is enhanced that has generated new electron avalanche in front of the head of streamer, and the electron attracted to the head of positive charge of streamer, then the channel of streamer strengthen and extension and the tails of positively ions are composed the positively charge of the head of streamer. Streamer and the head of positively charge made the electric field advanced and promoted the further development of streamer channel, then gradually advance toward the cathode.

When the rod has a negative polarity, the strong electric field region produce large amounts of electronic avalanche of rod electrode which join in the positive space charge around the rod electrode and the plasma present the diffusion layer distribution, which weaken the front electric field. At the period of elevation of voltage range, ionization is developed only between the rod electrode and the space of plasma layer epitaxy. When the electric field of plasma layer is strong enough, new electronic avalanche will develop and positive charge strengthen the front electric field of plasma layer, which formed a large number of secondary electron avalanche and made plasma layer push near the anode.

\section{c) Using ANSYS to simulate the rod plate gap}

The gap of rod and plate is set to $10 \mathrm{~mm}$, positive voltage is $20 \mathrm{kV}$, the negative voltage is $-20 \mathrm{kV}$, and the simulation is shown in Figure I,II:

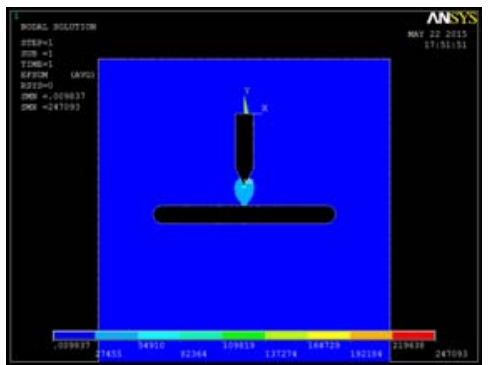

FIGURE I. POSITIVE ROD AND NEGATIVE PLATE

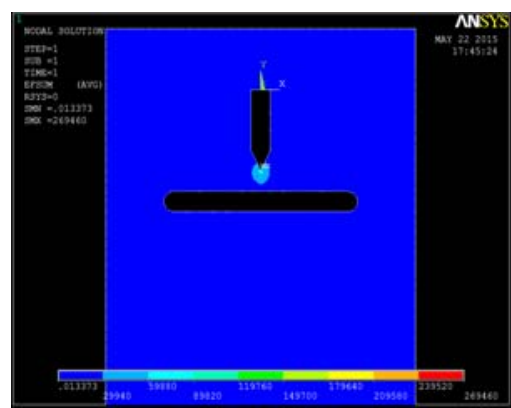

FIGURE II. NEGATIVE ROD AND POSITIVE PLATE

2) Experimental results show that:

a) When the rod is a positive polarity, the corona inception voltage is slightly higher than negative, that is $U+$ (corona) $>$ $\mathrm{U}$ - (corona). When the rod is a positive polarity, the space left by positive charge will cause the rod electrode surface electric field strength difficult to maintain. Once discharge occurs and the electron avalanche appears which developed from the maximum electric field at the rod electrode surface. The electric field is stronger, and the electron avalanche is more easily to appear. It is difficult to maintain self-sustaining after the electron avalanche occurs, because the electron avalanche will reduce the strength of electric field. So discharge could not be realized. It has to increase the voltage to meet the requirement of maintaining self-sustained discharge and corona discharge. That is the description of the initial voltage of discharge. Therefore, when the rod is a positive polarity, the corona inception voltage is slightly higher than negative.

b) When the rod is a negative polarity, the breakdown voltage is higher than the positive polarity, that is $U+$ (breakdown) $<\mathrm{U}$ - (breakdown). When voltage is applied, it is opposite of corona and breakdown. If the positive half cycle breakdown, the electric field will weaken. It is difficult to occur corona when the electric field is strengthen. To form streamer is equal to extend the rod electrode. If the streamer moved forward that is easy to be breakdown. So the corona voltage of rod electrode should be higher but the breakdown voltage should be lower.

\section{TyPiCAL InSULATION DEFECT ElECTROMAGNETIC SIMULATION}

In this paper, ANSYS is used to make simulation analysis of electric field distribution of several partial discharge defect models. Feasibility can be determined based on the simulation results of the electric field distribution of the defect physical model, and the result can be referenced for the following test portion. Two-dimensional physical defect model is used to simulate the distribution of the electric field, SF6 is set as solving domain, simulation boundary conditions is set as direct to ground, physical model parameters is shown in Table I, portions of the dielectric constant of the material is shown in Table II, cavity model is shown in Figure III.

TABLE I. TECHNICAL PARAMETERS OF MODELS

\begin{tabular}{ccc}
\hline $\begin{array}{c}\text { Thickness of } \\
\text { cavity }\end{array}$ & $\begin{array}{c}\text { Diameter of } \\
\text { cavity }\end{array}$ & $\begin{array}{c}\text { Diameter of } \\
\text { conductive rod }\end{array}$ \\
\hline 5 & 68 & 5 \\
\hline
\end{tabular}


TABLE II. THE RELATIVE PERMITTIVITY OF THE DIELECTRIC

\begin{tabular}{ccccccc}
\hline Material & SF6 & Al & $\begin{array}{c}\text { Stainless } \\
\text { steel }\end{array}$ & $\begin{array}{c}\text { Epoxy } \\
\text { resin }\end{array}$ & $\begin{array}{c}\text { Cooper } \\
\text { cuttings }\end{array}$ & Polytef \\
\hline $\begin{array}{c}\text { Relative } \\
\text { permittivity }\end{array}$ & 1.002 & 1.0 & 1.0 & 3.8 & 8000 & 2.55 \\
\hline
\end{tabular}

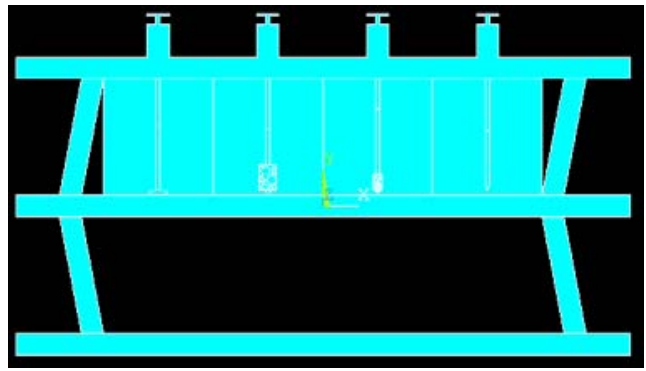

FIGURE III. BODY CAVITY AND DEFECT MODEL

\section{A. Simulation results of Suspended discharge}

Suspended potential is consisted of copper cake thickness of $0.8 \mathrm{~cm}$ and the diameter of $4 \mathrm{~cm}$. The top of copper cake is connected with PTFE insulated rod that diameter is $1 \mathrm{~cm}$ and length is $15 \mathrm{~cm}$. The top of PTFE insulated rod is connected with copper which is connected to the outdoor gas auger. The simulation result is shown in Figure IV. It is visible that near the stainless steel and bus, electric field appeared to be distorted, and electric field strength has been distorted near the suspension. When the voltage is applied to the model, partial discharge phenomenon is firstly appeared at the suspension. That is to say, the defect model is effective.

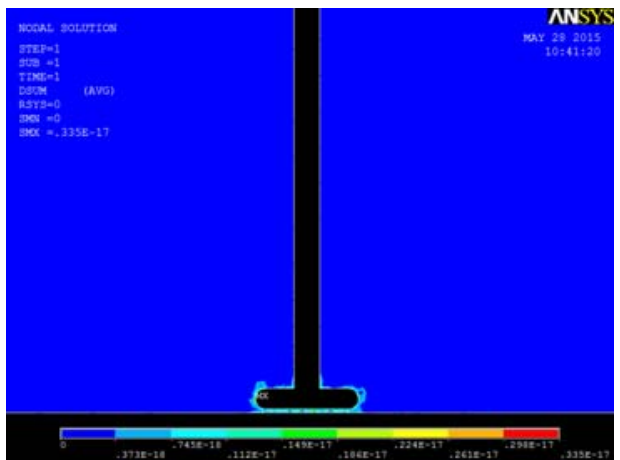

FIGURE IV. SIMULATION RESULTS OF SUSPENDED DISCHARGE

\section{B. Simulation results of insulators bubbles}

Insulators bubbles are consisted of epoxy resin with diameter of $4 \mathrm{~cm}$ and the length of $6 \mathrm{~cm}$. There are a lot of bubbles in epoxy resin. The bottom of the epoxy resin is connected with copper cylinder whose length is $1 \mathrm{~cm}$ and diameter is $1 \mathrm{~cm}$, and the top of epoxy resin is connected with copper cylinder whose length is $8 \mathrm{~cm}$ and diameter is $1 \mathrm{~cm}$. The top of copper cylinder is connected with copper which is connected to the outdoor gas auger. Actually, the distribution of model bubbles irregular, so, the simulation bubbles are set randomly. The relative permittivity of the bubbles are set to 1that is the constant of air. The simulation result shown in Figure V. It is visible that the strongest electric field strength is the bubbles near the conducting rod and easily to develop partial discharge.

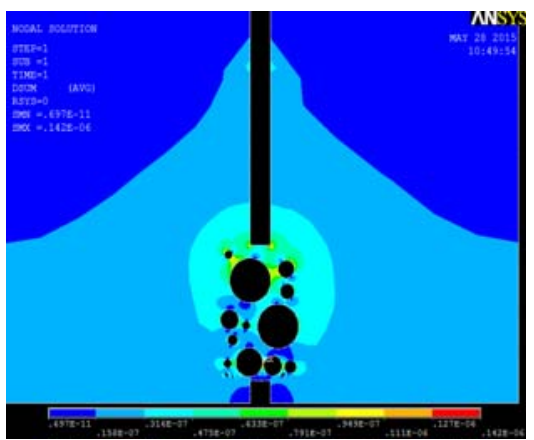

FIGURE V. SIMULATION RESULTS OF INSULATORS BUBBLES

\section{Simulation results of Metal particles}

Metal particles are composed by thirty copper balls. The diameter of five copper balls is $0.3 \mathrm{~cm}$ and the diameter of other twenty-five is $0.1 \mathrm{~cm}$. The metal particles have been put into hemispherical glass with $3 \mathrm{~cm}$ length and $2 \mathrm{~cm}$ diameter. The top of glass is connected with PTFE insulated rod that the diameter is $1 \mathrm{~cm}$ and length is $15 \mathrm{~cm}$. The top of PTFE insulated rod is connected with copper which is connected to the outdoor gas auger. The simulation result is shown in Figure VI. It is shown in picture that the electric field strength has been distorted near the metal particles. When the voltage is applied to the model, partial discharge phenomenon is firstly appeared at the metal particles. So the defect model is proven to be effective.

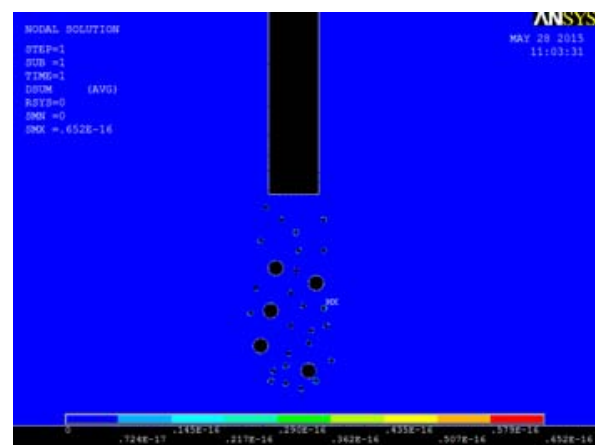

FIGURE VI. SIMULATION RESULTS OF METAL PARTICLES

\section{Simulation Result of Protrusion}

Protrusion is consisted of copper that the length of tip is $1.5 \mathrm{~cm}$, and the bottom diameter of the cone is $1 \mathrm{~cm}$. The top of copper cone is connected with copper cylinder which length is $12 \mathrm{~cm}$ and diameter is $1 \mathrm{~cm}$. The top of copper cylinder is connected with copper which is connected to the outdoor gas auger. The outside boundary of body cavity is set as direct to ground, and the technical parameters are set as the experimental model. The simulation result is shown in Figure VII. It is shown that the electric field strength has been distorted seriously near the protrusion.

The simulation results show that: 
a) The initial discharge voltage values of defect models are less than $140 \mathrm{kV}$ and the discharge phenomenon of GIS test is due to the defect model that placed inside the cavity. The phenomenon of the defect model is the basis of the following experiment.

b) The initial discharge voltage and discharge capacity of different defect models are not the same. Discharge capacity is vary from a large gap of the different defect models, and the damage to GIS is different.

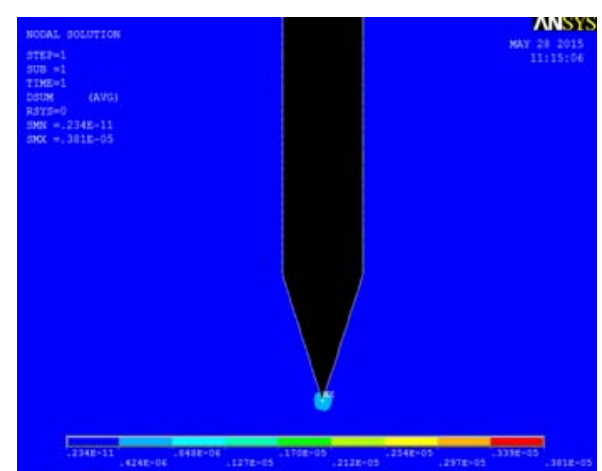

FIGURE VII. SIMULATION RESULT OF PROTRUSION

\section{EXPERIMENT SET}

The device can simulate a variety of partial discharge phenomenon of $220 \mathrm{KV}$ GIS. The equipment can provide the real controllable partial discharge site for pulse current method, UHF method, Ultrasound method and other testing research, evaluation and testing equipment.

\section{A. Partial Discharge Test Equipment}

Test platform rely on the GIS test equipment of Hangzhou Xihu Institute of Electronic Research High-voltage Laboratory. The model of GIS is based on the partial discharge defect structure, and the test GIS is shown in Figure VIII. The test equipment GIS are constituted by AC power control box, power isolation filter, GIS and a built-in transformer. Auxiliary ventilation equipments are consisted by rotary vane vacuum pump and the tank of SF6. Then SF6 gas is charged into GIS to $0.3 \mathrm{MPa}$.

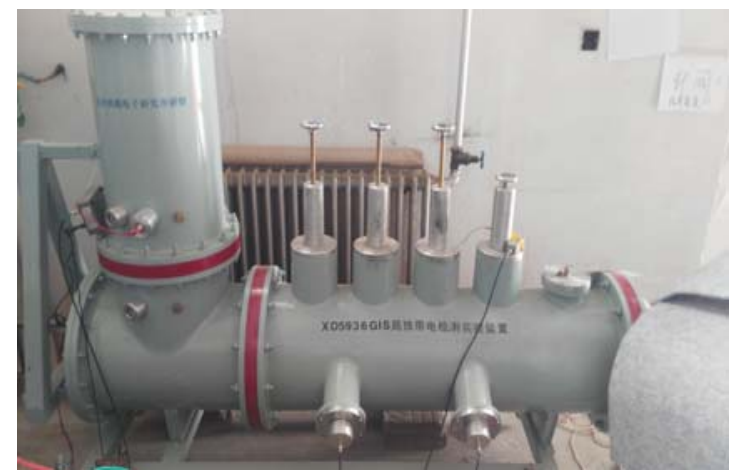

FIGURE VIII. EXPERIMENTAL GIS

There are three chambers of equipment, as shown in the picture, the left side of the chamber is the bus experimental bus chamber and the right side of the chamber is the transformer chamber. Experimental gas chamber can put four kinds of defects at the same time, and the height of defects can be adjusted up and down through the auger to selecting the experimental defects. The camera is used to monitor the type and distance of defects monitoring, and at the head of left cylinder there is the UHF sensor.

Test schematic is shown in Figure IX, and the hardware can be divided into external pressure regulating device and GIS insulation defect simulation test device.

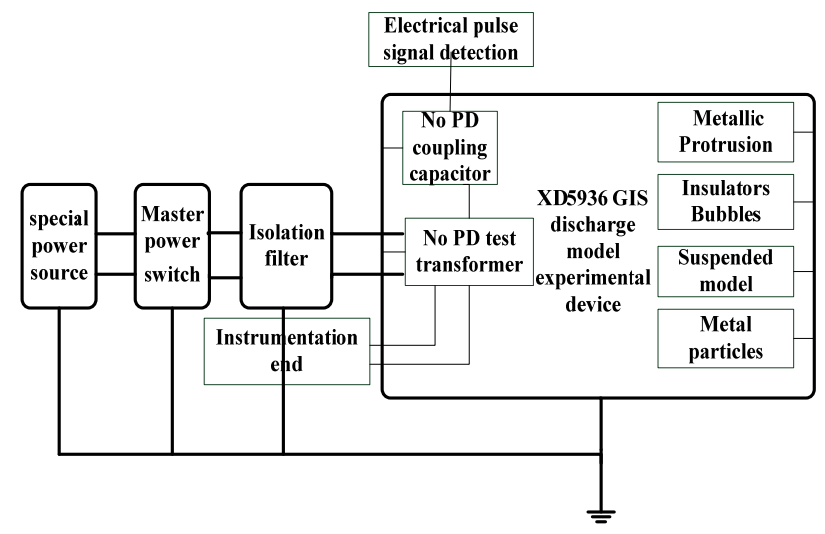

FIGURE IX. TEST SCHEMATIC

\section{B. Partial discharge detection system}

Detection systems are consists of UHF sensors, ultra-high frequency signal receiver, $\mathrm{AD}$ acquisition card and computer operating interface and so on. UHF sensor selects the design of cavity-backed spiral antenna, and it has a relatively flat frequency response in the frequency range from $300 \mathrm{MHz}$ to $2000 \mathrm{MHz}$, and the VSWR (voltage standing wave ratio) is less than 2. UHF receiver is consists by a pre-filter, low-noise amplifier, mixer, IF filter and signal processor, etc. The UHF detection system is shown in Figure $\mathrm{X}$, and the final frequency is selected range from $400 \mathrm{MHz}$ to $1600 \mathrm{MHz}$.

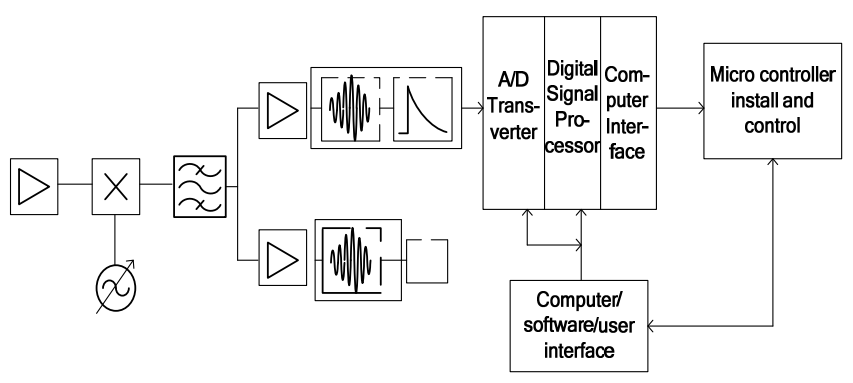

FIGURE X. UHF DETECTION SYSTEM

The traditional method of pulse current is recommended by IEC60270 standard. When partial discharge occurs, the charge of the test loop will change and the current develope. The conditions of partial discharge can be detected by measuring the pulse voltage across the detection resistor, and this is called pulse current method. This method is more suitable for laboratory measurements, and the traditional pulse current principle is shown in Figure XII. 


\section{Typical defect model}

In order to guarantee the same partial discharge data of the experiment and the real partial discharge site, this passage simulates the same typical defects of the real. The experiment simulates four types of defects, suspended, insulators bubbles, metal particles and protrusion. Experimental model is shown in Table III of left and the monitoring screen of right.

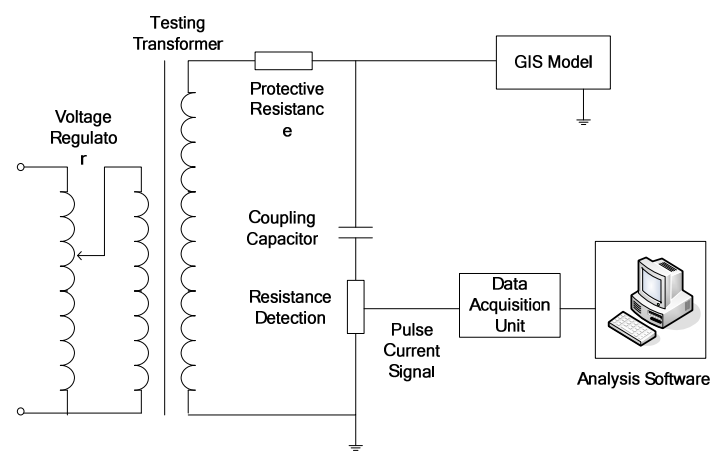

FIGURE XI. PULSE CURRENT DETECTING SCHEMATIC

TABLE III. FOUR DEFECT MODELS

\begin{tabular}{|l|l|l|}
\hline \multicolumn{1}{|c|}{ Defect type } & Defect model & \multicolumn{2}{|c|}{ Monitor } \\
\hline Suspended & & \\
\hline $\begin{array}{l}\text { Insulators } \\
\text { bubbles }\end{array}$ & & 1 \\
\hline $\begin{array}{l}\text { Metal } \\
\text { particles }\end{array}$ & & 1 \\
\hline $\begin{array}{l}\text { Metallic } \\
\text { Protrusion }\end{array}$ & & 1 \\
\hline
\end{tabular}

D. Testing experiment

1) The PD test of cavity pressure level

The voltage is increased step by step - to $160 \mathrm{kV}$, and experimental measurement of cavity PD level is $6 \mathrm{pC}$, less than maximum $7 \mathrm{pC}$. The reason why $\mathrm{PD}$ is big is that the poor conditions of grounding.

\section{2) The background test of UHF}

By contrast test and influence of high-frequency signals, the sensitivity of built-in UHF sensor are tested effectively. It is shown that the built-in sensor has a better detection performance.

\section{3) The statistics of initial voltage of typical defect}

The statistics of initial voltage of typical defect is shown in Table IV.

TABLE IV. THE INITIAL DISCHARGE VOLTAGE OF DEFECT

\begin{tabular}{|c|c|c|c|}
\hline \multicolumn{2}{|c|}{ Defect type } & UHF(kV) & Pulse Current(kV) \\
\hline \multirow{2}{*}{ Suspended } & $0.5 \mathrm{~mm}$ & 51.7 & 55 \\
\cline { 2 - 4 } & $1.5 \mathrm{~mm}$ & 80 & 97.2 \\
\hline $\begin{array}{c}\text { Insulators } \\
\text { bubbles }\end{array}$ & $0 \mathrm{~mm}$ & 25.55 & 30 \\
\hline \multirow{2}{*}{$\begin{array}{c}\text { Metal } \\
\text { particles }\end{array}$} & $2 \mathrm{~mm}$ & 42.4 & 50.47 \\
\cline { 2 - 4 } & $6 \mathrm{~mm}$ & 75.6 & 80.13 \\
\hline $\begin{array}{c}\text { Metallic } \\
\text { Protrusion }\end{array}$ & $1 \mathrm{~cm}$ & 29.17 & 35.5 \\
\cline { 2 - 4 } & $2 \mathrm{~cm}$ & 37.40 & 52.2 \\
\hline
\end{tabular}

The tests show that:

It can be seen through the test that the UHF method can firstly detect the discharge signals that distinguished from background, so the UHF method is more sensitive than the pulse current method.

The initial voltage of the defect far from bus is larger than that near the bus. Because the greater distance to the bus, the stronger gas-insulated. Once the discharge occurs, there is greater amount of discharge.

The initial voltage of the suspended defect model is the maximum of the four kinds of the defect. When the discharge happened, the discharge capacity is quite large. Even in the initial voltage of the discharge phenomenon, there will be a spark discharge and a "bang, bang" sound. It is shown that the suspended defect was particularly serious damage to the GIS. Once the suspended defect happened, it is highly probable to generate the insulation breakdown failure.

\section{4) The feature extraction of $P D$}

The UHF method is used to detect the different internal defects of GIS of the test. And the characteristic parameters are selected from the pulse phase distribution mode (Phase Resolved Partial Discharge, PRPD) pattern, as shown in Figure XII.

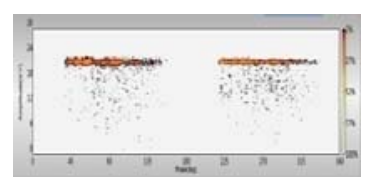

(a) Suspended defect of PRPD

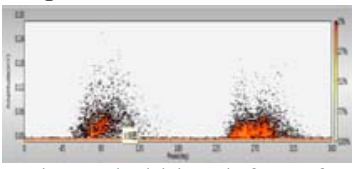

(c) Insulators bubbles defect of PRPD

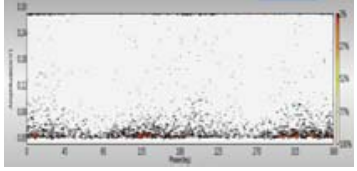

(e) Metal particles defect of PRPD

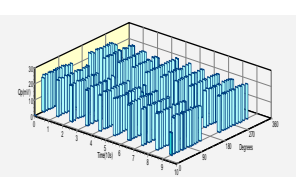

(b) 3D Pattern

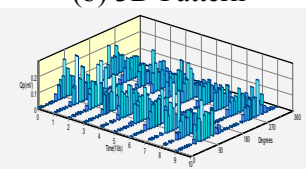

(d) 3D Pattern

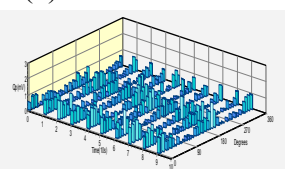

(f) 3D Pattern 


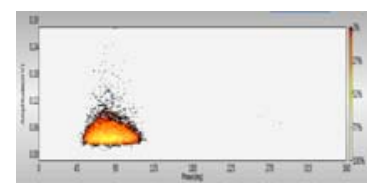

(g) Metallic Protrusion defect of PRPD

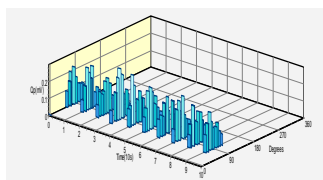

(h) 3D Pattern

FIGURE XII. CHARACTERS OF FOUR TYPES OF DEFECT PD SOURCES

As shown in Figure (a) (b), the suspended defect discharge is detected both in the positive and negative half-wave, and the phase characteristics of the discharge are obvious that the magnitude maintain at a certain value. As shown in Figure (c) (d), the insulator bubbles are detected both in the positive and negative half-wave, and the discharge is divided into two layers, that the discharge is distributed of the bottom phase and the phases of the upper are obvious. As shown in Figure (e) (f) the metal particles have no obvious defect phase discharge characteristics, and the whole cycle of the phases are occurred to discharge. As shown in Figure (g) (h), the protrusion defect discharge appeared in the positive half-wave, and the discharge phase characteristics are obvious that the discharge shaped like a cone.

\section{4) The dimension reduction of the selected data}

Using the basic idea of principal component analysis, through the linear weighted combination of original elements of " $\mathrm{m}$ " and constitute new elements of " $\mathrm{r}$ " that is mutually orthogonal $(r \leqslant m ; r, m \in N)$ and the main characteristics of the original can be saved.

According to the cumulative variance contribution rate to select the dimension, and the rate often selected between $85 \%$ and $90 \%$. The main components of the contribution rate of the first four are shown in Table V.

TABLE V. THE CUMULATIVE CONTRIBUTION RATE

\begin{tabular}{ccccc}
\hline Main components & PCA1 & PCA2 & PCA3 & PCA4 \\
\hline Accumulative contribution rate & 0.40 & 0.65 & 0.84 & 0.90 \\
\hline
\end{tabular}

\section{PARTIAL Discharge FAUlt TyPE IDENTIFICATION}

\section{A. System Architecture}

The structure of ANFIS that proposed for the GIS fault diagnosis is complex. Four-dimensional data is selected as the input vector that the original characteristics is preserved $90 \%$. The first layer is the fuzzy layer which parameters can be automatically adjusted by the learning network, and the amount of membership functions is 12 . The second layer is a regular layer, and each neuron corresponds with a single Sugeno type fuzzy rules. The inputs of neurons of rules are received from the neurons of fuzzy, and the strength of rules is calculated. The domain space can be completely covered by 81 nodes that used by the layer. The third layer is normalized layer. The inputs of neurons of normalized layer are received from the neurons of rules, and the normalized degree of activation could be calculated under a given rule. The forth layer is the de-fuzzy layer. The inputs of neurons of de-fuzzy layer are received from the neurons of normalized layer and the initial input is R1, R2, R3, R4. The fifth layer is a sum of the neurons, and the neurons compute the sum of all de-fuzzy neurons then output the final results of ANFIS. The original data are divided into four groups for training and four groups for testing and there are ten data samples for each group. The data was trained in accordance with predetermined parameters. Training error is 0.00013 , and the error curve is smoothly.

\section{B. PD pattern recognition results}

After training the ANFIS, the test data samples are put into the model to verify. The test results are shown in Figure XIII, and 1 represents the suspended defect type, 2 represents the insulator bubbles defect type, 3 represents the metal particles defect type, 4 represents the protrusion defect type, represents the desired output and * represents the actual output of ANFIS.

The identification results are shown in the FIGURE XIII, the actual output of ANFIS is basically met the requirements, and the average test error is 0.076 . This system is effective that it is feasible that the ANFIS fuzzy neural network is used to identify the defect type of insulation fault GIS.

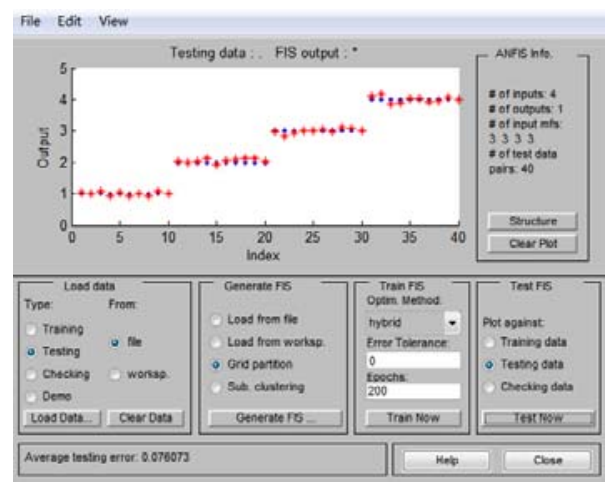

FIGURE XIII. IDENTIFICATION RESULTS

\section{CONCLUSIONS}

In this paper, four conclusions are concluded.

a) ANSYS is used to simulate the defect models and qualitatively analyze the partial discharge which is caused by defect model rather than the device itself.

b) Several experiments of the real site are summarized in this paper that the initial discharge voltage and the discharge capacity are different from the types of the defect models.

c) Principal component analysis is used to reduce the dimension of the original data that $90 \%$ of the characteristics are saved and the subsequent data processing is reduced.

d) ANFIS is used to distinguish the four types of the defect models that the recognition accuracy is high and it is feasible as the method of defect identification.

\section{ACKNOWLEDGMENT}

This work was supported by Inner Mongolia Natural Science Foundation under grant 2015MS0543 and by Graduate Natural Science Foundation of Inner Mongolia under grant S20141012805. 


\section{REFERENCES}

[1] QI Bo, LI Cheng-rong, HAO Zhen, et al. Evolution phenomena and features of surface partial discharge initiated by immobilized metal particles on GIS insulators[J]. Proceedings of the CSEE, 2011, 31(1): 101-108.

[2] WANG Hui, HUANG Cheng-jun, YAO Lin-peng, et al. Time-frequency Analysis of Partial Discharge for GIS Using the Theoty of Reassignment Distribution[J].High Voltage Engineering, 2010, 36(9): 2236-2241.

[3] LIN Li-bo. The applied research of Partial Discharge Monitoring System for Gas Insulated Switchgear[D]. Guangzhou: South China University of Technology, 2011.

[4] DING Deng-wei, GAO Wen-sheng, LIU Wei-dong. Analysis on the typical partial discharge using UHF detection method for GIS[J]. High Voltage Engineering, 2011,37(3): 706-710.

[5] QIAN Yong, HUANG Cheng-jun, JIANG Xiu-chen, et al. Current status and development of PD online monitoring technology in GIS[J]. High Voltage Apparatus, 2004, 40(6): 453-456.

[6] SONG Bei-hua. Based on the UHF and combined GIS pratial discharge on-line detection technology research[D]. Shanghai: Shanghai Jiaotong University, 2012.

[7] QIU Yu-chang. Use UHF method on-line monitoring in GIS insulation[J]. High Voltage Apparatus, 1997(4): 36-40.

[8] OKABES, YAMAGIWA T, OKUBO H. Detection of harmful metallic particles inside gas insulated switchgear using UHF sensor[J]. IEEE Transactions on Dielectrics and Electrical Insulation, 2008, 15(3): 701709.

[9] Luo Li-shi, Yao Wen-jun, Wang jun and so on. Research on Partial Discharge Diagnosis of GIS by Decomposed Gas of SF_6 [J]. Power System Technology,34 (5): 225-230, 2010.

[10] Yan Xiang-lian, Wang Cheng-yu, Yang Ren and so on . Fault Diagnosis of High Voltage Switchgears by Decomposition Products of SF_6 [J] Power System Technology,35 (12): 118-123, 2011.

[11] Zhang Xiao-xing, Yao Yao, Tang Ju . Actuality and Perspective of Proximate Analysis of SF 6 Decomposed Products Under Partial Discharge [J]. High Voltage Enngineering,34 (4): 664-669 + 747, 2008.

[12] Wang Xian-han, Wang Feng, Qiu Yu-chang. Dynamic Simulations of Two-dimensional Streamer Discharge in Uniform Field in SF_6 [J]. High Voltage Engineering,34 (7): 1358-1362, 2008.

[13] Zhang Yun, Zeng Rong, Li xiao ling .Numbering Simulation on Streamer Discharge of Short Air Gap of Atmospheric Air[J]. Proceedings of the CSEE, 2008, 28(28): 6-12.

[14] Zhang Yun, Zeng Rong,Yang Xue-chang . Study on Photoionization Produced by Discharge in Atmospheric Air by Numerical Method [J]. Proceedings of the CSEE, 2009, 29(4): 110-116.

[15] Tang Ju. Partial discharge recognition based on SF_6 decomposition products and support vector machine[J]. IET Science, Measurement and Technology, 6(4): 198-204, 2012.

[16] Zhang Xiaoxing .GIS Partial discharge Pattern Recognition based on the Chaos Theory[J]. IEEE , 21(2): 2014

[17] Jyh-Shing Roger Jang. Adaptive-Network-Based Fuzzy Inference System [J]. IEEE , 1993.

[18] Liu Wei, Su Hong-sheng, Zeng Xiao-qin . Diagnosis of Transformer Faults Based on Improved ANFIS [J]Chongqing Institute of Technology (Natural Science),04: 107-111, 2009. 